\title{
THE ARITHMETICITY OF A KODAIRA FIBRATION IS DETERMINED BY ITS UNIVERSAL COVER
}

\author{
GABINO GONZÁLEZ-DIEZ AND SEBASTIÁN REYES-CAROCCA
}

\begin{abstract}
Let $S \rightarrow C$ be a Kodaira fibration. Here we show that whether or not the algebraic surface $S$ is defined over a number field depends only on the biholomorphic class of its universal cover.
\end{abstract}

\section{INTRODUCTION AND STATEMENT OF RESUlTS}

Let $X \subset \mathbb{P}^{n}$ be a complex projective variety and $k$ a subfield of the field of the complex numbers $\mathbb{C}$. We shall say that $X$ is defined over $k$ or that $k$ is a field of definition for $X$ if there exists a collection of homogenous polynomials $f_{0}, \ldots, f_{m}$ with coefficients in $k$ so that the variety they define is isomorphic to $X$. We will say that $X$ is arithmetic if it is defined over $\overline{\mathbb{Q}}$ or equivalently over a number field.

While it is classically known that there are only three simply connected Riemann surfaces, there is a huge amount of possibilities for the holomorphic universal cover of a complex surface $S$. It would be interesting to understand the extent to which the arithmeticity of a projective surface can be read off from its holomorphic universal cover. In this short note we study this question for a very important class of complex surfaces known in the literature as Kodaira fibrations.

A Kodaira fibration consists of a non-singular compact complex surface $S$, a compact Riemann surface $C$ and a surjective holomorphic map $S \rightarrow C$ everywhere of maximal rank such that the fibers are connected and not mutually isomorphic Riemann surfaces. The genera $g$ of the fibre and $b$ of $C$ are called the genus of the fibration and of the base respectively. It is known that such a surface $S$ must be an algebraic surface of general type and that necessarily $g \geq 3$ and $b \geq 2$. We notice that an important theorem by Arakelov [1] implies that, up to isomorphism, there are only finitely many Kodaira fibrations over a given algebraic curve $C$.

In 1967, Kodaira [13] used fibrations of this kind to show that the signature of a differentiable fiber bundle need not be multiplicative. Soon after Kas [12] studied the deformation space of the surfaces constructed by Kodaira, and two years later Atiyah [2] and Hirzebruch [10] studied further

2000 Mathematics Subject Classification. 32J25, 14J20, 14J25.

Key words and phrases. Complex surfaces and their universal covers, Field of definition.

The authors were partially supported by Spanish MEyC Grant MTM 2012-31973. The second author was also partially supported by Becas Chile. 
properties concerning the signature of Kodaira fibrations in a volume dedicated to Kodaira himself.

Explicit constructions of Kodaira surfaces have been made by GonzálezDiez and Harvey 8, Bryan and Donagi [4, Zaal [16 and Catanese and Rollenske [6].

We now state the main results of the paper

Theorem 1. Let $k$ be an algebraically closed subfield of the complex numbers and $S_{1} \rightarrow C_{1}$ and $S_{2} \rightarrow C_{2}$ two Kodaira fibrations so that their respective holomorphic universal covers are biholomorphically equivalent. Then $S_{1}$ is defined over $k$ if and only if $S_{2}$ is defined over $k$. In particular, $S_{1}$ is arithmetic if and only if $S_{2}$ is arithmetic.

To prove this theorem we will have to show first the following result which is interesting in its own right

Theorem 2. Let $k$ be an algebraically closed subfield of the complex numbers and $S \rightarrow C$ a Kodaira fibration. Then $S$ is defined over $k$ if and only if $C$ is defined over $k$. In particular, $S$ is an arithmetic surface if and only if $C$ is an arithmetic curve.

Theorem 1 states that the arithmeticity of a Kodaira fibration can be recognized in its holomorphic universal cover. We anticipate that the holomorphic universal cover of $S$ is a contractible bounded domain $\mathscr{B} \subset \mathbb{C}^{2}$ (see Section 2). Clearly, Theorem 1 implies that the biholomorphism class of $\mathscr{B}$ varies together with the variation of $S$ in moduli space. We note that in general Kodaira surfaces are not rigid ([12], [6]).

\section{Uniformization of Kodaira Surfaces}

It is well-known that the universal cover of a Riemann surface is biholomorphically equivalent to the projective line $\mathbb{P}^{1}$, the complex plane $\mathbb{C}$ or the upper half-plane $\mathbb{H}$. Understanding universal covers of complex manifolds of higher dimension seems to be a very complicated task. However, thanks to the work of Bers [3] and Griffiths [9] on uniformization of algebraic varieties, it is possible to describe the universal cover of a Kodaira fibration $f: S \rightarrow C$ in a very explicit way.

Let $\pi: \mathbb{H} \rightarrow C$ be the universal covering map of $C$ and $\Gamma$ the covering group so that $C \cong \mathbb{H} / \Gamma$. By considering the pull-back $h: \pi^{*} S \rightarrow \mathbb{H}$ of $f$ by $\pi$, we obtain a new fibration in which, for each $t \in \mathbb{H}$, the fiber $h^{-1}(t)$ agrees with the Riemann surface $f^{-1}(\pi(t))$. Teichmüller theory enables us to choose uniformizations $h^{-1}(t)=D_{t} / K_{t}$ possessing the following properties:

(a) $K_{t}$ is a Kleinian group acting on a bounded domain $D_{t}$ of $\mathbb{C}$ which is biholomorphically equivalent to a disk.

(b) The union of all these disks $\mathscr{B}:=\cup_{t \in \mathbb{H}} D_{t}$ is a contractible bounded domain of $\mathbb{C}^{2}$ which is biholomorphic to the universal cover of $S$, that is, $S \cong \mathscr{B} / \mathbb{G}$, where $\mathbb{G}<\operatorname{Aut}(\mathscr{B})$ is the covering group. 
(c) The group $\mathbb{G}$ is endowed with a surjective homomorphism of groups $\Theta: \mathbb{G} \rightarrow \Gamma$ which induces an exact sequence of groups

$$
1 \longrightarrow \mathbb{K} \longrightarrow \mathbb{G} \stackrel{\Theta}{\longrightarrow} \Gamma \longrightarrow 1
$$

where, for each $t \in \mathbb{H}$, the subgroup $\mathbb{K}$ preserves $D_{t}$ and acts on it as the Kleinian group $K_{t}$.

We note that $\mathscr{B}$ carries itself a fibration structure $\mathscr{B} \rightarrow \mathbb{H}$ whose fiber over $t \in \mathbb{H}$ is $D_{t}$ (i.e. $\mathscr{B}$ is a Bergman domain in Bers' terminology).

In 14 and 15. Shabat studied the automorphism groups of universal covers of families of Riemann surfaces and proved a deep result which in the case of Kodaira fibrations amounts to the following theorem.

Theorem (Shabat) Let $f: S \rightarrow C$ be a Kodaira fibration and $\mathscr{B}$ the holomorphic universal cover of $S$. Then:

(a) the covering group $\mathbb{G}$ of $S$ has finite index in $\operatorname{Aut}(\mathscr{B})$.

(b) $\operatorname{Aut}(\mathscr{B})$ is a discrete group.

\section{Proof of Theorems 1 and 2}

We denote by $\operatorname{Gal}(\mathbb{C})$ the group of field automorphisms of $\mathbb{C}$. The natural action of $\operatorname{Gal}(\mathbb{C})$ on the ring of polynomials $\mathbb{C}\left[x_{0}, \ldots, x_{n}\right]$ induces a well-defined action $(\sigma, X) \rightarrow X^{\sigma}$ on the set of isomorphism classes of algebraic varieties. Throughout this section $k$ will denote an algebraically closed subfield of $\mathbb{C}$ and $\operatorname{Gal}(\mathbb{C} / k)$ the subgroup of $\operatorname{Gal}(\mathbb{C})$ consisting of all automorphisms $\sigma$ fixing the elements of $k$.

3.1. Proof of Theorem 2, Let $f: S \rightarrow C$ be a Kodaira fibration. Let us assume that the curve $C$ is defined over $k$. Then $C^{\sigma}=C$ for all $\sigma \in$ $\operatorname{Gal}(\mathbb{C} / k)$, and so, by Arakelov's finiteness Theorem, there are only finitely many pairwise non-isomorphic Kodaira fibrations $f^{\sigma}: S^{\sigma} \rightarrow C^{\sigma}$ with $\sigma \in$ $\operatorname{Gal}(\mathbb{C} / k)$. This implies that $S$ is defined over $k$ [7, Crit. 2.1].

In order to prove the converse, we begin by recalling that a complex manifold $X$ is named hyperbolic if every holomorphic map $\mathbb{C} \rightarrow X$ is a constant map. We claim that Kodaira fibrations are hyperbolic. In fact, let $f: S \rightarrow C$ be a Kodaira fibration and $\varphi: \mathbb{C} \rightarrow S$ a non-constant holomorphic map. As $C$ has genus greater than one, the map $f \circ \varphi: \mathbb{C} \rightarrow C$ must be constant and therefore the image of $\varphi$ has to be contained in a fiber $f^{-1}(x)$ for some $x \in C$. Since the fibers are also hyperbolic, $\varphi$ must be constant too.

Let us now assume that $S$ is defined over $k$, so that $S^{\sigma}=S$ for any $\sigma \in \operatorname{Gal}(\mathbb{C} / k)$. Now as $S$ is a Kähler hyperbolic manifold, the canonical divisor $K_{S}$ is ample [5] and this implies that only finitely many curves $R$ of genus greater than one can arise as the image of a surjective morphism $S \rightarrow R$ [1]. In particular the family $\left\{C^{\sigma}: \sigma \in \operatorname{Gal}(\mathbb{C} / k)\right\}$ itself contains only finitely many isomorphism classes of curves. It then follows that $C$ is defined over $k$ [7, Crit. 2.1], as required. 
3.2. Proof of Theorem 1. Let $f_{2}: S_{2} \rightarrow C_{2}$ be a Kodaira fibration and $S_{1}$ an arbitrary non-singular complex surface. Let us denote by $\mathscr{B}_{i}$ the universal cover of $S_{i}$ and suppose that there exists an isomorphism $\alpha$ : $\mathscr{B}_{1} \rightarrow \mathscr{B}_{2}$ between them. Let $\mathbb{G}_{i}$ be the uniformizing group of $S_{i}$ so that $\mathscr{B}_{i} / \mathbb{G}_{i} \cong S_{i}$. By Shabat's Theorem $\mathbb{G}_{2}$ has finite index in $\operatorname{Aut}\left(\mathscr{B}_{2}\right)$. We claim that $\mathbb{G}_{1}$ has finite index in $\operatorname{Aut}\left(\mathscr{B}_{1}\right)$ too. In fact, as $\mathscr{B}_{1} / \operatorname{Aut}\left(\mathscr{B}_{1}\right) \cong$ $\mathscr{B}_{2} / \operatorname{Aut}\left(\mathscr{B}_{2}\right)$ and as $\operatorname{Aut}\left(\mathscr{B}_{2}\right)$ is a discrete group, the projection map $S_{1}=$ $\mathscr{B}_{1} / \mathbb{G}_{1} \rightarrow \mathscr{B}_{1} / \operatorname{Aut}\left(\mathscr{B}_{1}\right)$ is a holomorphic map between (normal) compact complex surfaces; from here the claim follows.

By replacing $\mathbb{G}_{1}$ by $\alpha \mathbb{G}_{1} \alpha^{-1}$ we can assume that $\mathscr{B}_{1}=\mathscr{B}_{2}$, so we denote $\mathscr{B}_{i}$ simply by $\mathscr{B}$. As both $\mathbb{G}_{1}$ and $\mathbb{G}_{2}$ have finite index in $\operatorname{Aut}(\mathscr{B})$, so must do their intersection $\mathbb{G}_{12}=\mathbb{G}_{1} \cap \mathbb{G}_{2}$. The complex surface $S_{12}:=\mathscr{B} / \mathbb{G}_{12}$ is endowed with two finite degree covers $\pi_{i}^{\prime}: S_{12} \rightarrow S_{i}$ with $i=1,2$; in particular, $S_{12}$ is also a projective surface. Moreover, if we denote by $\Theta_{12}$ the restriction of the epimorphism $\Theta: \mathbb{G}_{2} \rightarrow \Gamma_{2}$ introduced in the previous section to $\mathbb{G}_{12}$, then we obtain an exact sequence of groups

$$
1 \longrightarrow \mathbb{K}_{12} \longrightarrow \mathbb{G}_{12} \stackrel{\Theta_{12}}{\longrightarrow} \Gamma_{12} \longrightarrow 1
$$

where $\Gamma_{12}=\Theta_{12}\left(\mathbb{G}_{12}\right)$ and $\mathbb{K}_{12}=\operatorname{ker}\left(\Theta_{12}\right)=\mathbb{K} \cap \mathbb{G}_{12}$. As in Section 2, this sequence defines a Kodaira fibration $f_{12}: S_{12} \rightarrow C_{12}:=\mathbb{H} / \Gamma_{12}$ whose fiber over $[t]_{\Gamma_{12}}$ is isomorphic to the Riemann surface $D_{t} / K_{t}^{12}$ where $K_{t}^{12}$ is the Kleinian group that realizes the action of $\mathbb{K}_{12}$ on $D_{t}$. We have the following commutative diagram

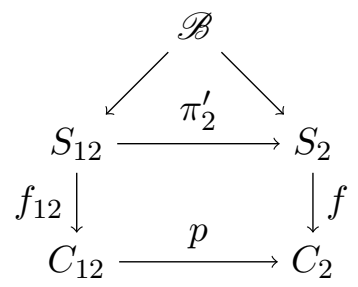

where $p$ is the projection induced by the finite index inclusion $\Gamma_{12}<\Gamma_{2}$.

Let us now assume that $S_{2}$ is defined over $k$. Then Theorem 2 ensures that $C_{2}$ is also defined over $k$. Furthermore, being an unbranched cover of $C_{2}$, the curve $C_{12}$ must also be defined over $k$ [7, Th. 4.1]. Again, by Theorem 2 we conclude that $S_{12}$ is defined over $k$. Now, as $S_{1}$ is a surface of general type arising as the image (by $\pi_{1}^{\prime}$ ) of a surface defined over $k$, it must be defined over $k$ as well [7, Prop. 3.2]. This proves Theorem 1.

\section{REFERENCES}

[1] S. Arakelov, Families of curves with fixed degenerancies, Math. USRR, Izvestija, 5, 1277-1302, (1971).

[2] M. Aтіуан, The signature of fiber-bundles, Global Analysis (Papers in Honor of K. Kodaira), University of Tokyo Press, (1969), 73-84.

[3] L. BeRs, Uniformization, moduli and kleinian groups, London Math. Soc., 4, (1972), 257-300. 
[4] J. Bryan And R. Donagi, Surface bundles over surfaces of small genus, Geom. Topol., 6, (2002), 59-67.

[5] F. CAMPanA, Twistor spaces and non-hyperbolicity of certain symplectic Kähler manifolds, Complex analysis, Aspects of Mathematics, 1, (1991), 64-69.

[6] F. Catanese and S. Rollenske, Double Kodaira Fibrations, J. Reine Angew. Math. 628 (2009), 205-233.

[7] G. GonzÁlez-Diez, Variations on Belyi's Theorem, Quart. J. Math. 57, (2006), 339-354.

[8] G. GonzÁlez-Diez And W. Harvey, On complete curves in moduli space I, Math. Proc. Cambridge Philos. Soc., 110 (1991), no.3, 461-466.

[9] P. A. GRIfFiths, Complex analytic properties of certain Zariski open sets on algebraic varieties, Ann. of Maths., 94 (1971), 21-51.

[10] F. Hirzebruch, The signature of ramified coverings, Global Analysis (Papers in Honor of K. Kodaira), University of Tokyo Press, (1969), 253-265.

[11] A. Howard And A. J. Sommese, On the theorem of de Franchis, Annali della Scuola Normale Superiore di Pisa, 4 serie, tome 10, 3, (1983), 429-436.

[12] A. KAs, On deformations of a certain type of irregular algebraic surfaces, J. Analyse Math., 19 (1967), 207-215.

[13] K. Kodaira, A certain type of irregular algebraic surfaces, Amer. J. Math., 90 (1968), 789-804.

[14] G. Shabat, The complex structure of domains covering algebraic surfaces, Funct. Anal. Appl. 11, (1977), 135-142.

[15] G. SHABAT, Local reconstruction of complex algebraic surfaces from universal coverings, Funktsional. Anal. i Prilozhen. 17 (1983), no.2, 90-91.

[16] C. ZAAL, Explicit complete curves in the moduli space of curves of genus three, Geom. Dedicata, 56, (1995), no.2, 185-196.

Departamento de Matemáticas, Universidad Autónoma de Madrid.

E-mail address: gabino.gonzalez@uam.es, sereyesc@gmail.com 